\title{
Primary Tuberculous Stricture of Splenic Flexure of Colon
}

\author{
Authors \\ Girish D. Bakhshi, Kavita V. Jadhav, Mohd. Siddique JA, Pankajkumar J. Zanwar, \\ Jasmine R. Agarwal, Mukund B. Tayade
}

Department of General Surgery, Grant Govt Medical College \& Sir J.J.Group of Hospitals, Mumbai-400008 ABSTRACT
Abdominal tuberculosis is an increasingly common disease that poses diagnostic challenge as the
nonspecific features of the disease may lead to diagnostic delays and the development of complications.
Tuberculosis of gastrointestinal tract commonly affects distal ileum and ileocaecal junction. Segmental
colonic tuberculosis is uncommon and constitute $9.2 \%$ of all abdominal tuberculosis. We are presenting a
case of primary tuberculous stricture of splenic flexure which posed a diagnostic dilemma.
KEYWORDS: primary tuberculous stricture, splenic flexure of transverse colon

\section{INTRODUCTION}

Tuberculosis of gastrointestinal tract commonly affects ileum \& ileocaecal junction. Segmental primary tuberculosis of colon is uncommon. It usually presents as sub-acute intestinal obstruction. It is important to suspect and diagnose tuberculosis early to avoid surgical intervention as it responds very well to anti tubercular treatment (ATT). It needs surgical intervention if here is complication in the form of obstruction or perforation. In present case, patient had recurrent sub-acute intestinal obstruction and malignant stricture of splenic flexture was preliminary diagnosis. However, it turned out to be tuberculous stricture of splenic flexure of transverse colon.

\section{CASE REPORT}

A 35 years old woman presented with recurrent symptoms of constipation, vomiting, intermittent pain in abdomen, loss of weight and abdominal distension. Patient had no history suggestive of tuberculosis in past.

Clinical examination revealed mild abdominal distension without any guarding and rigidity. Patient had no lump or organomegaly. Ultrasonography (USG) was suggestive of distended large bowel loops with normal peristalsis. Computed tomography with contrast (CECT) abdomen was suggestive of high grade stricture in transverse colon at splenic flexure with gross dilatation of caecum, ascending and transverse colon (Fig.1\& 2). Tumour marker carcino embryonic antigen (CEA) was found within normal limit. Colonoscopy was done, which revealed circumferential stricturous thickening at splenic flexure.

Biopsy was taken and specimen was sent for histopathological examination (HPE). HPE report was suggestive of benign stricture without evidence of malignancy.

As patient was having grossly distended bowel 
loops and recurrent attacks of sub-acute obstruction, exploratory laparotomy with left hemicolectomy with lymph node dissection and colo-colic anastomosis was done. Histopathology was suggestive of tuberculous stricture of transverse colon with reactive lymph nodes.

Patient recovered well in post-operative period and was discharge on post-op day 10 on ATT (3 months of Isoniazid + Rifampicin + Ethambutol + Pyrizinamide and 6 months of Isoniazid + rifampicin). Patient followed up for 1 year, and found to be symptom and disease free.

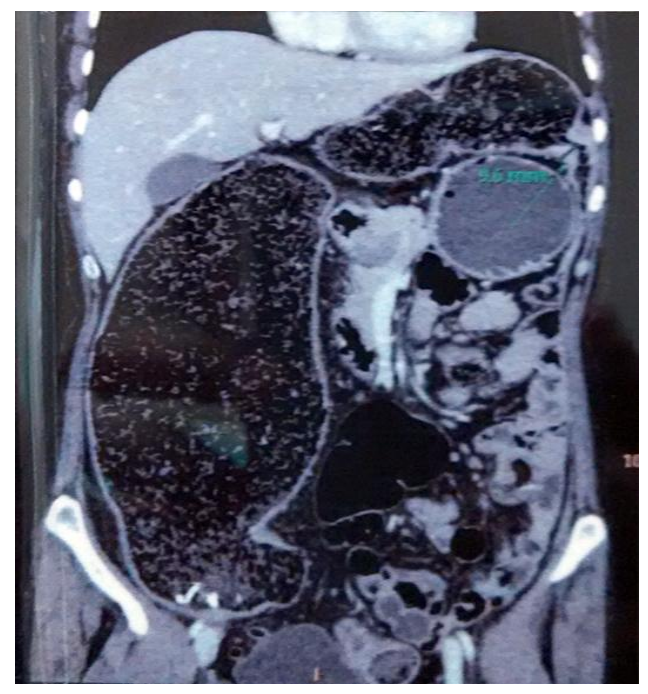

Fig.1 - CT coronal view showing grossly dilated caecum, ascending and transverse colon with benign stricture at splenic flexure

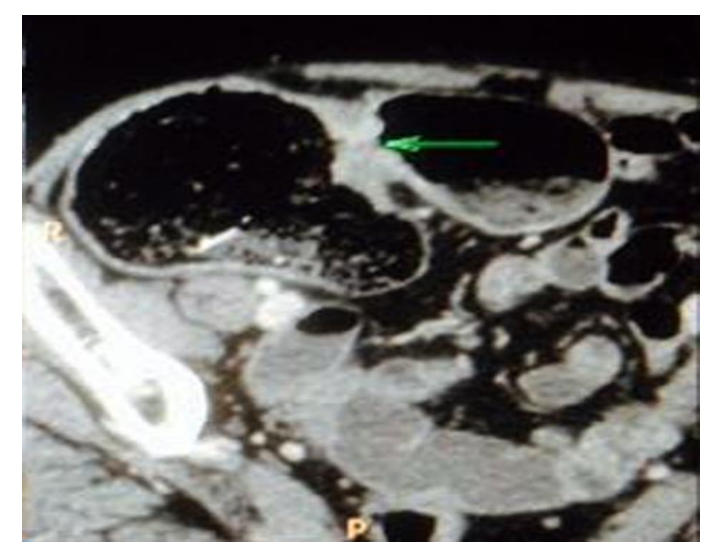

Fig. 2 - CT Axial view showing stricturous segment in splenic flexure of colon

\section{DISCUSSION}

Tuberculosis is known to involve any segment of gastrointestinal tract. Tuberculosis of the small bowel is common. Isolated primary tuberculosis of the large bowel is uncommon. It constitutes 9.2 $\%$ of all abdominal tuberculosis ${ }^{1}$. Ascending colon is commonly involved along with ileocaecal junction. It usually presents as subacute or acute intestinal obstruction. Most of the times surgeon suspects malignancy as the cause of colonic stricture.

On imaging like ultrasonography, Barium studies and computed tomography, we can easily diagnose stricturous growth, but colonscopy plays a vital role in diagnosis. Biopsy taken with the help of colonoscopy may diagnose segmental tuberculosis. ATT can be started as conservative line of management. Those who don't respond to ATT, usually require surgical management.

In present case, patient had recurrent episodes of subacute intestinal obstruction (SAIO). On USG \& CT scan, malignant stricture of splenic flexure was kept as first diagnosis. Colonscopy was suggestive of splenic flexure stricturous growth. biopsy was suggestive of benign stricture. Since, patient had recurrent SAIO, decision to explore the patient was taken. Intraoperatively, grossly dilated transeverse colon with stricture at splenic flexture was found. Mesenteric lymphadeopathy was present. Left hemicolectomy along with lymph node desection with colo-colic anastomosis was performed. Histopathology turned out to be tuberculosis of colon. Patient had no history or history suggestive of tuberculosis. Primary tuberculous stricture of splenic flexure is rare.

Haque \& Siddiqui et $\mathrm{al}^{2}$ have presented case reports of 2 patients with segmental colonic tuberculosis. In this report, one patient had tubercular splenic flaxure stricture. Left hemicolectomy with colocolic anastomosis was done in that case. Other patient had hepatic flexure stricturous growth and on investigations diagnosed as tuberculosis but patient failed to respond to ATT and surgery was performed in that patient. 


\section{CONCLUSION}

Though gastrointestinal tuberculosis is common, primary tubercular stricture of splenic flexure of transeverse colon is uncommon. High index of suspicion is essential for early diagnosis and treatment for prevention of complications. During evaluation of colonic strictures along with neoplastic etiology, possibility of benign causes like tuberculosis should be kept in mind, specially in Indian subcontinent.

\section{REFERANCES}

1. Chawla S, Mukerjee P, Bery K. Segmental tuberculosis of the colon: a report of ten cases. Clin Radiol 1971;22:104-9.

2. Haque M , Siddique A, Badruddoza S , Amin M, Rahman M , Hasan M, Abedin M. Primary Segmental Colonic Tuberculosis: A Case Report .TAJ: The Journal of Teachers Association. 2004;17:52-4. 